\title{
artigo
}

\section{Programas de rastreamentos dos valores da pressão arterial no mundo: revisão integrativa}

\author{
Programs for screening blood pressure values in the world: an integrative review \\ Programas para rastrear los valores de la presión arterial en el mundo: una revisión integrativa
}

\begin{abstract}
RESUMO
Objetivo: analisar as evidências disponíveis na literatura sobre os programas de rastreamentos dos valores da pressão arterial no mundo. Método: revisão integrativa da literatura, realizada no mês de setembro de 2019 com artigos no período de 2015 a 2019, nas bases de dados National Library of Medicine National Institutes of Health, Literatura latino-americana e do Caribe em ciências da saúde, Web of Science, no Banco de Dados Scopus e na Biblioteca Cochrane. Resultados: Um total de 13 estudos foi selecionado para integrar esta revisão. Rastreamentos da pressão arterial realizados ressaltam a importância do mesmo para conscientização, detecção precoce, controle e prevenção de valores da pressão elevadores. Conclusão: Programa de rastreamento da pressão arterial é uma estratégia de baixo custo e elevada eficácia, para identificação do diagnóstico, tratamento e controle da hipertensão arterial, seja em países desenvolvidos e em desenvolvimento, e ao mesmo tempo contribui para diminuição de riscos para doenças cardiovasculares.

DESCRITORES: Determinação da Pressão Arterial; Programas de Rastreamento; Hipertensão; Pressão Arterial; Enfermagem.
\end{abstract}

\section{ABSTRACT}

Objective: to analyze the evidence available in the literature on the programs of tracing two values of arterial pressure in the world. Method: integrative literature review, carried out no more than September 2019 with the period from 2015 to 2019, based on data bases National Library of Medicine National Institutes of Health, Latin American and Caribbean literature in health science, Web of Science, not Scopus Dice Bank in the Cochrane Library. Results: A total of 13 studies were selected to integrate this review. Traces of arterial pressure carried out to highlight the importance of the same for awareness, early detection, control and prevention of elevating pressure values. Conclusion: Arterial pressure monitoring program is a low-custodial strategy and high efficiency, for the identification of diagnosis, treatment and control of arterial hypertension, according to developed and developing countries, and at the same time it contributed to the reduction of risks for cardiovascular diseases.

DESCRIPTORS: Blood Pressure Determination; Mass Screening; Hypertension; Blood Pressure; Nursing.

\section{RESUMEN}

Objetivo: analizar como evidências disponíveis en la literatura sobre los programas de rastreamentos dos valores de la presión arterial en el mundo. Método: revisão integrativa da literatura, realizada no mês de setembro de 2019 com artigos no período de 2015 a 2019, nas bases de dados National Library of Medicine National Institutes of Health, Literatura latino-americana e do Caribe em ciências da saúde, Web of Science, no Banco de Dados Scopus e na Biblioteca Cochrane. Resultados: Um total de 13 estudos seleccionados para integrar esta revisión. Rastreamentos da pressão arterial realizó ressaltam a importância do mesmo para conscientização, detecção precoce, control e prevenção de valores da pressão elevadores. Conclusión: Programa de rastreamento da presão arterial é uma estratégia de baixo custo e elevada eficácia, para identificação do diagnóstico, tratamento e controle da hipertensão arterial, seja em países desenvolvidos e em desenvolvimento, e ao mesmo tempo contribui para diminuição de riscos para doenças cardiovasculares.

DESCRIPTORES: Determinación de la presión arterial; Tamizaje Masivo; Hipertensión; Presión arterial; Enfermería.

RECEBIDO EM: 11/11/2020 APROVADO EM: 23/11/2020

\section{Bárbara Caroliny Pereira Costa}

Enfermeira. Universidade de São Paulo, Escola de Enfermagem de Ribeirão Preto, Ribeirão Preto, SP, Brasil.

ORCID: 0000-0003-3945-5240 


\section{Victoria Garibalde Hilario}

Graduanda em enfermagem. Universidade de São Paulo, Escola de Enfermagem de Ribeirão Preto, Ribeirão Preto, SP, Brasil. ORCID: 0000-0001-6467-3122.

\section{Eugenia Velludo Veiga}

Enfermeira. Universidade de São Paulo, Escola de Enfermagem de Ribeirão Preto, Ribeirão Preto, SP, Brasil.

ORCID: 0000-0003-3677-0210

\section{INTRODUÇÃO}

A s Doenças Crônicas Não Transmissíveis (DCNT) têm apresentado um aumento significativo nos últimos anos, sendo as mais comuns, a Hipertensão Arterial (HA), as Doenças Cardiovasculares $(\mathrm{DCV})$, as respiratórias crônicas e o Diabetes Mellitus. Essas constituem a primeira causa de mortalidade no mundo, correspondendo a $70 \%$ das mortes e no Brasil a porcentagem chega a $75 \%{ }^{(1)}$.

Esse crescimento das DCNT tem ocorrido devido ao aumento de fatores de risco modificáveis como, uso prejudicial do álcool e tabaco, inatividade física e alimentação não saudável ${ }^{(2)}$. Tais fatores de risco também estão relacionados principalmente ao aumento da HA, que acrescentam em seus fatores de risco, a idade, sexo e etnia, excesso de peso e obesidade, ingestão de sal, fatores socioeconômicos e genética ${ }^{(2-3)}$.

A HA caracteriza-se por uma condição clínica multifatorial, sustentada pela elevação dos níveis pressóricos $\geq 140 \mathrm{mmHg}$ pressão sistólica e/ou $90 \mathrm{mmHg}$ pressão diastólica, sendo uma das principais causas responsáveis pela morbimortalidade em todo o mundo. Tem sido considerada como principal fator de risco global e importante problema de saúde pública. No Brasil, atinge $32,5 \%$ da população adulta e mais de $60 \%$ dos idosos, e contribui com $50 \%$ dos óbitos por DCV, de forma direta ou indireta. Essa doença crônica sustenta uma relação independente com eventos como acidente vascular encefálico (AVE), infarto agudo do miocárdio (IAM), insuficiência cardíaca (IC), morte súbita, dentre outros, as quais são complicações bastante ocorridas ${ }^{(2,4)}$.

A HA pode ser associada a distúrbios metabólicos, alterações funcionais e/ou estruturais de órgãos-alvo, além de ser agra- vada pela a existência de fatores de risco como, idade avançada; sexo e etnia; excesso de peso e obesidade; consumo excessivo de sal; Diabetes Mellitus; fatores socioeconômicos; assim como, fatores genéticos ${ }^{(3)}$.

\section{A HA caracteriza-se} por uma condição clínica multifatorial, sustentada pela elevação dos níveis pressóricos $\geq 140$ mmHg pressão sistólica e/ou 90 mmHg pressão diastólica, sendo uma das principais causas responsáveis pela morbimortalidade em todo o mundo.

A eficácia no diagnóstico e tratamento da HA é por meio da medida correta da Pressão Arterial (PA), muitas vezes considerada padrão ouro de diagnóstico em medicina convencional. Esse procedimento é uma das técnicas mais realizadas pelos profissionais da saúde e principalmente pe- los da enfermagem, é considerado simples e o método é fácil, mas requer cuidados para obtenção de resultados fidedignos ${ }^{(3,5)}$.

Realiza-se a medida da PA para o rastreio da HA, na qual utiliza-se de iniciativas como os Programas de Rastreamento da Pressão Arterial, que é de baixo custo e contribui com o diagnóstico antecipado HA, auxilia no controle da doença e objetiva reduzir a morbimortalidade das doenças cardiovasculares em diferentes países ${ }^{(6)}$.

Dessa maneira, o enfermeiro assume papel fundamental neste contexto, uma vez que ele detém conhecimentos e habilidades para realizar o rastreamento e promover ações de educação em saúde à população para adoção de melhores hábitos de vida ${ }^{(7)}$. Estes devem atuar de forma eficaz no rastreamento e no processo de educação em saúde à população. Essas ações possuem como principal objetivo a detecção precoce da HA, a adaptação do paciente à doença, a prevenção de complicações, incluindo sua adesão ao tratamento e acompanhamento médico, assim o indivíduo se torna agente de seu autocuidado, reduzindo a mortalidade ${ }^{(8)}$. Assim, o objetivo do presente estudo foi analisar as evidências disponíveis na literatura sobre os programas de rastreamentos dos valores da pressão arterial no mundo.

\section{MÉTODO}

Trata-se de uma revisão integrativa da literatura, a qual tem como finalidade identificar e analisar as evidências científicas de forma sistemática e ordenada, sobre o conhecimento do tema pesquisado. Para o rigor metodológico do estudo, seis etapas foram percorridas ${ }^{(9)}$.

Em cumprimento a primeira etapa da revisão integrativa foi utilizada a estratégia 
PICO, que trata-se de uma ferramenta utilizada pela prática baseada em evidências e é representa pelo acrônimo dos termos em inglês "Patient", "Intervention", "Comparion" e "Outcomes". Tal estratégia foi aplicada na fase inicial desta revisão como contribuição à elaboração da questão da pesquisa e devido à necessidade de se identificar palavras-chaves para a localização de estudos relevantes nas bases de dados selecionadas ${ }^{(10)}$.

Neste estudo, o P refere-se ao paciente/ problema: Rastreamento da pressão arterial; o I como Intervenção ou indicador: como tem sido a realização do rastreamento da pressão arterial no mundo. O C, como Comparação ou controle, neste caso não se aplica, e $\mathrm{O}$ como desfecho: verificar se há disponível na literatura estudos que tem realizado o rastreamento da pressão arterial. Mediante a estratégia PICO a seguinte questão norteadora foi formulada para a presente revisão: Como tem sido realizado os programas de rastreamento da pressão arterial no mundo?

Foram incluídos no estudo artigos publicados na íntegra, no período de 2015 a 2019 e redigidos em português, inglês ou espanhol e excluídos artigos duplicados, relatos de experiência, teses, dissertações ou artigos cuja temática eram incompatíveis com a proposta deste estudo.

A busca bibliográfica foi realizada no mês de setembro de 2019 nas bases de dados National Library of Medicine National Institutes of Health (PuBMed), Literatura latino-americana e do Caribe

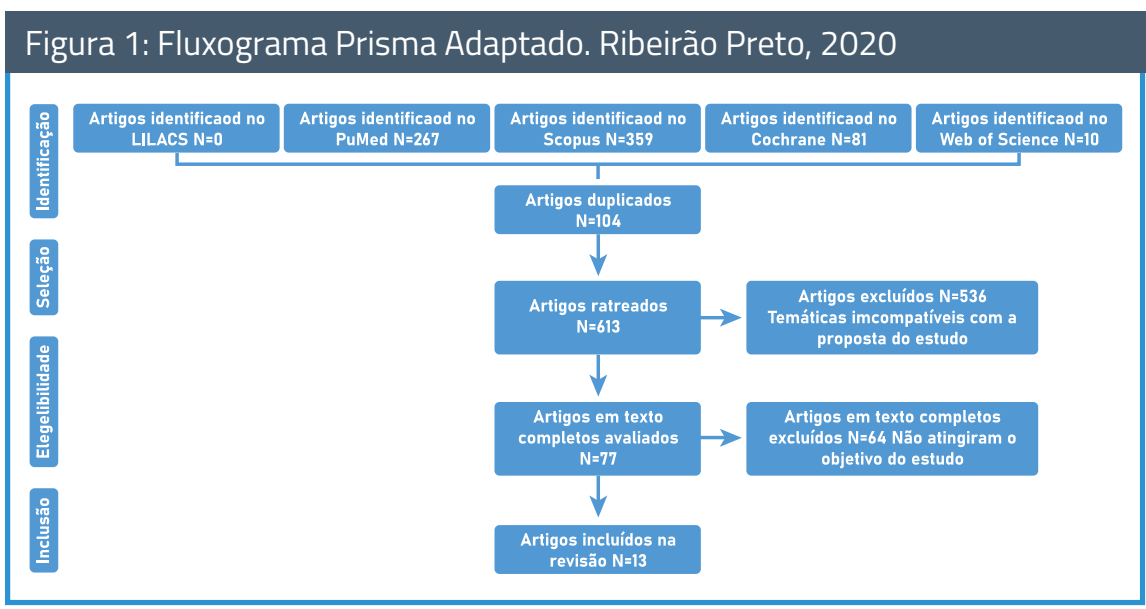

em ciências da saúde (LILACS), Web of Science, no Banco de Dados Scopus e na Biblioteca COCHRANE. Os Descritores em Ciências da Saúde (DeCS) da Biblioteca Virtual de Saúde e descritores em língua inglesa Medical Subject Headings (MeSH) utilizados na busca foram: Determinação da Pressão Arterial (Blood Pressure Determination), Pressão Arterial (Blood Pressure), Hipertensão (Hypertension) e Programas de Rastreamento (Mass Screening) com a aplicação do operador booleano "AND" e "OR" entre os termos.

$\mathrm{Na}$ fase de triagem dos artigos foi utilizado um programa de rastreio designado por Rayyan ${ }^{\circ(11)}$. Este permite a otimização das revisões de literatura. $\mathrm{O}$ programa mostra o título, resumo, data de publicação dos textos carregados das bases de dados para que o pesquisador os analise e permite a opção de inclusão ou exclusão, além de uma aba designada como "talvez", caso o pesquisador tenha dúvida na inclusão e queira revisar o devido artigo. Ao final, o programa permite uma organização da revisão, na qual o pesquisador saberá quantos artigos foram excluídos, incluídos e duplicados.

Após a seleção dos artigos por meio do programa Rayyan', foi utilizada a estratégia PRISMA para promover o relato da presente revisão. A recomendação PRISMA consiste em um checklist com 27 itens e um fluxograma de quatro etapas com intuito de ajudar os autores a melhorarem o relato de revisões sistemáticas e meta-análises ${ }^{(12)}$. A Figura 1 ilustra as para integrar esta revisão, sendo três (23\%) de Coorte, 8 (61, 5\%) com abordagem quantitativa e dois $(15,5 \%)$ descritivos.

Em relação à origem dos estudos, apenas um $(7,6 \%)$ foi desenvolvido no Brasil, os outros 12 estão distribuídos entre China (7,6\%), Israel (7,6\%), Malawi (7,6\%), Estados Unidos (7,6\%) África do Sul (7,6\%), Índia (7,6\%), dois (15,3\%) na Itália, dois (15,3\%) Inglaterra e dois $(15,3 \%)$ estudos são multicêntricos, na qual um foi realizado na França e Marrocos e outro programa de rastreamento que envolveu 80 países diferentes. Todos foram publicados no idioma inglês (100\%). 
No tocante aos níveis de evidência, 10 estudos foram classificados como nível VI (77\%) e 3 (23\%) foram classificados como nível IV, o que sugere que grande parte dos artigos analisados apresentaram níveis de evidência considerados fracos, indicando a necessidade de desenvolvimento de es- tudos com níveis de evidencia mais forte, capaz de subsidiar práticas seguras e eficazes ${ }^{(12)}$. O Quadro 1 descreve os artigos selecionados na amostra conforme título, autores, ano de publicação, objetivo principal, tipo de estudo e principais resultados/conclusão.

\section{DISCUSSÃO}

Estudos de rastreamento da pressão arterial realizados em vários países ressaltam a importância do mesmo para conscientização, detecção precoce, controle e prevenção de valores da pressão arterial eleva-

Quadro 1 - Artigos selecionados conforme autores, ano de publicação, objetivo principal, tipo de estudo e principais resultados.

\begin{tabular}{|c|c|c|}
\hline AUTORES/ ANO & OBJETIVO/TIPO DE ESTUDO & RESULTADOS \\
\hline $\begin{array}{l}\text { Omboni S, Verberk } \\
\text { WJ. } 2019^{(15) .}\end{array}$ & $\begin{array}{l}\text { Testar se um monitor de pressão } \\
\text { automáatico oscilométrico que permite a } \\
\text { medição simultânea da pressão arterial } \\
\text { entre os braços. Quantitativo }\end{array}$ & $\begin{array}{l}\text { Indivíduos com diferença significativa de pressão arterial anormal entre } \\
\text { braços foram entre pessoas mais velhas, que apresentaram maior índice } \\
\text { de massa corporal, maiores níveis de PA e foram mais propensos a re- } \\
\text { latar obesidade, história de HA ou DCV do que indivíduos com diferença } \\
\text { de pressão entre braços normal. A medição simultânea da PA em ambos } \\
\text { os braços deve ser realizada em indivíduos com risco para ou com DCV } \\
\text { estabelecida. }\end{array}$ \\
\hline $\begin{array}{l}\text { Balsari S. et al. } \\
2017^{(16)}\end{array}$ & $\begin{array}{c}\text { Realizar um programa de rastreamento } \\
\text { de HA. Quantitativo }\end{array}$ & $\begin{array}{l}\text { No Kumbh, } 5760 \text { pessoas optaram voluntariamente pelo rastreamento } \\
\text { da HA e receberam uma única medida da pressão arterial. No total, } 1783 \\
\text { apresentaram um rastreamento positivo, dos quais } 1580 \text { desconheciam } \\
\text { previamente seu diagnóstico; } 55 \text { (18\%) tiveram leituras normais da } \\
\text { pressão arterial. Estudo demonstra que o rastreamento comunitário } \\
\text { para HA não costuma consumir muitos recursos, precisa de pouca } \\
\text { infraestrutura e treinamento e geralmente é econômica. }\end{array}$ \\
\hline $\begin{array}{l}\text { Goldberg E. M. et } \\
\quad \text { al. } 2017(17)\end{array}$ & $\begin{array}{l}\text { Avaliar a confiabilidade da pressão } \\
\text { arterial do rastreamento de emergência } \\
\text { e determinar se a identificação correta } \\
\text { varia de acordo com gênero, raça ou } \\
\text { qualidade da mensuração. Coorte }\end{array}$ & $\begin{array}{l}\text { Dos } 354 \text { pacientes de unidade de emergência participaram do estudo } \\
\text { com idade mediana de } 39 \text { anos, sendo } 48,9 \% \text { mulheres, } 66,4 \% \text { brancos } \\
\text { e } 17 \% \text { negros. De todos os participantes } 66,1 \% \text { apresentaram resulta- } \\
\text { dos da pressão arterial } \geq 120 / 80 \text { mmHg.. Dois terços dos pacientes } \\
\text { sem diagnóstico de } H A \text { apresentaram pressão arterial acima do limiar de } \\
120 / 80 \text { mmHg em rastreamento. }\end{array}$ \\
\hline $\begin{array}{l}\text { Beaney T. et al. } \\
2018^{(18)}\end{array}$ & $\begin{array}{l}\text { Aumentar a conscientização sobre os } \\
\text { valores da pressão arterial elevados. } \\
\text { Quantitativo, transversal }\end{array}$ & $\begin{array}{l}\text { Foram coletados dados de } 1.201 .570 \text { indivíduos em } 80 \text { países. Desses, } \\
34,9 \% \text { indivíduos apresentavam hipertensão. } 17,3 \% \text { de } 888.616 \text { indiví- } \\
\text { duos que não estavam recebendo tratamento anti-hipertensivo eram } \\
\text { hipertensos e } 105.456 \text { ( } 46,3 \% \text { ) dos } 227.721 \text { indivíduos que receberam } \\
\text { tratamento não tinham pressão arterial controlada. A campanha identi- } \\
\text { ficou mais de } 335.000 \text { adultos com HA controlada e não controlada não } \\
\text { tratada ou inadequadamente tratada. }\end{array}$ \\
\hline $\begin{array}{l}\text { Bar-Dayan Y. et } \\
\text { al. } 2016^{(19)}\end{array}$ & $\begin{array}{l}\text { Avaliar a eficácia de uma operação de } \\
\text { rastreamento de disglicemia, diabetes e } \\
\text { alterações na pressão arterial em uma } \\
\text { estação de saúde e ambiente público. } \\
\text { Quantitativo, transversal }\end{array}$ & $\begin{array}{l}13.112 \text { adultos foram submetidos ao rastreamento, dos quais } 16,9 \% \\
\text { relataram diabetes e } 23,2 \% \text { hipertensão. Já entre os que não tinham co- } \\
\text { nhecimento de hipertensão } 9,6 \% \text { possuíam níveis pressóricos elevados } \\
(\geq 140 \text { / 90). O rastreamento pôde identificar indivíduos com altos níveis } \\
\text { de pressão arterial e risco de disglicemia que foram encaminhados para } \\
\text { diagnóstico e tratamento. }\end{array}$ \\
\hline $\begin{array}{l}\text { Handler, J. et al. } \\
2015^{(20)}\end{array}$ & $\begin{array}{l}\text { Identificar a prevalência e característi- } \\
\text { cas de pacientes com pressão arterial } \\
\text { elevada na atenção não- primária em } \\
\text { comparação com as visitas de cuidados } \\
\text { primários. Coorte }\end{array}$ & $\begin{array}{l}\text { Adultos normotensos com pelo menos } 12 \text { meses de adesão ao plano } \\
\text { de saúde em } 1 \text { de janeiro de } 2009 \text { ( } n=1.075 .522 \text { ) foram acompanha- } \\
\text { dos para PA elevada até } 14 \text { de março de } 2011 \text {. Dos } 111.996 \text { pacientes } \\
\text { com } P A \geq 140 \text { / } 90 \mathrm{~mm} \mathrm{Hg}, 82,7 \% \text { foram medidos durante as visitas de } \\
\text { cuidados primários e } 17,3 \% \text { durante as visitas de cuidados não primários. }\end{array}$ \\
\hline $\begin{array}{l}\text { Kachimanga, C. et } \\
\quad \text { al } 2017^{(21)}\end{array}$ & $\begin{array}{l}\text { Analisar três programas de rastrea- } \\
\text { mento para HA e diabetes implantados } \\
\text { diretamente na comunidade de Neno, } \\
\text { Malawi. Descritivo }\end{array}$ & $\begin{array}{l}\text { Mais de } 14.000 \text { adultos ( } \geq 12 \text { anos) foram rastreados, dos quais } 58 \% \\
\text { foram rastreados para } \mathrm{HA} \text { e } 9 \% \text { encaminhados para avaliação adicional. } \\
\text { Desde o início do programa de rastreamento o número de pacientes em } \\
\text { tratamento de DCNT quase triplicou. }\end{array}$ \\
\hline
\end{tabular}




\section{artigo}

Costa, B.C.P.; Hilario, V.G.; Veiga, E.V.

Programas de rastreamentos dos valores da pressão arterial no mundo: revisão integrativa

\begin{tabular}{|c|c|c|}
\hline $\begin{array}{l}\text { Chen, S. et al. } \\
\text { 2019 }\end{array}$ & $\begin{array}{l}\text { Estimar o impacto do rastreamento da } \\
\text { pressão arterial baseado na comunidade } \\
\text { sobre os níveis subsequentes de pressão } \\
\text { arterial entre adultos mais velhos na } \\
\text { China. Coorte }\end{array}$ & $\begin{array}{l}\text { A amostra inicial foi composta por } 6010 \text { pessoas. Foram excluídas } 437 \\
\text { pessoas, e entre os } 5573 \text { participantes restantes, } 3899 \text { (70\%) tinham HA } \\
\text { não diagnosticada. A intervenção reduziu a pressão arterial sistólica: -6,3 } \\
\text { mmHg. O impacto na pressão arterial diastólica foi menor e não signifi- } \\
\text { cativo. }\end{array}$ \\
\hline $\begin{array}{l}\text { Poulter, N. R. et } \\
\text { al. } 2017^{(23)}\end{array}$ & $\begin{array}{l}\text { Destacar a importância da avaliação da } \\
\text { pressão arterial elevada. Descritivo }\end{array}$ & $\begin{array}{l}\text { Como resultado dos exames da campanha de rastreamento, pode-se } \\
\text { prever que um número significativo de novos adultos hipertensos será } \\
\text { detectado, e se a pressão arterial for reduzida em apenas } 10 \text { mmHg em } \\
\text { média, isso reduzirá a morbimortalidade cardiovascular em cerca de } 25 \% \text {. } \\
\text { É esperado que a campanha fortaleça a capacidade de causar um grande } \\
\text { impacto na prevenção, tratamento e controle da hipertensão mundial. }\end{array}$ \\
\hline $\begin{array}{l}\text { Destro, M. et al. } \\
2015^{(24)}\end{array}$ & $\begin{array}{c}\text { Avaliar a prevalência de HA e avaliar } \\
\text { outros fatores de risco cardiovasculares. } \\
\text { Quantitativo }\end{array}$ & $\begin{array}{l}\text { Entre maio de } 2011 \text { e maio de } 2014 \text {, } 1540 \text { indivíduos foram avaliados, } \\
890 \text { declarados normotensos e } 650 \text { declarados hipertensos. De todos, } \\
1137 \text { apresentaram PA <140/ } 90 \text { mmHg, entre eles } 408 \text { individuos } \\
\text { eram declarados hipertensos. Pressão Arterial > } 140 \text { / } 90 \text { mmHg foi ob- } \\
\text { servada em } 403 \text { indivíduos. Esses resultados representam maior risco } \\
\text { cardiovascular em indivíduos hipertensos do que normotensos. }\end{array}$ \\
\hline $\begin{array}{l}\text { Rheeder, P. et al. } \\
\qquad 2016^{(25)}\end{array}$ & $\begin{array}{l}\text { Melhorar a detecção e o manejo de } \\
\text { DCNT em ambientes urbanos e rurais } \\
\text { negligenciados. Quantitativo }\end{array}$ & $\begin{array}{l}\text { Foram rastreados } 7607 \text { pessoas, dos quais } 37,1 \% \text { apresentaram resul- } \\
\text { tados de pressão arterial em valor } \geq 140 \text { mmHg na PA sistólica e } \geq 90 \\
\text { mmHg na PA diastólica. } 8,3 \% \text { apresentaram niveis aleatórios de glico- } \\
\text { se capilar aleatórios } \geq 7,8 \mathrm{mmol} / \text { I. Ao final da pesquisa a procura dos } \\
\text { membros da comunidade em rastreamento por uma confirmação diag- } \\
\text { nóstica foi menor do que a esperada, o que mostrou pouca efetividade } \\
\text { da conscientização sobre as DCNT. }\end{array}$ \\
\hline $\begin{array}{l}\text { Risse, J. et al. } \\
2015^{(26)}\end{array}$ & $\begin{array}{c}\text { Avaliar uma estratégia de rastreamento } \\
\text { da pressão arterial elevada para pacien- } \\
\text { tes hipertensos que desconhecem o } \\
\text { diagnóstico ou não o realizam. Quanti- } \\
\text { tativo }\end{array}$ & $\begin{array}{l}\text { Participaram } 1011 \text { na França e } 299 \text { pessoas em Marrocos. Foi identi- } \\
\text { ficado uma porcentagem muito alta de pacientes hipertensos não tra- } \\
\text { tados em Marrocos }(70,2 \%) \text { e França }(25,7 \%) \text {. Na França, } 40,2 \% \text { dos que } \\
\text { apresentaram uma PA } \geq 135 / 85 \mathrm{mmHg} \text { procuraram um médico após o } \\
\text { rastreamento. }\end{array}$ \\
\hline $\begin{array}{l}\text { Jardim, T. et al. } \\
2015 \text { (27). }\end{array}$ & $\begin{array}{l}\text { Comparar as diferenças nas taxas de } \\
\text { prevalência, conscientização, tratamento } \\
\text { e controle da HA. Quantitativo }\end{array}$ & $\begin{array}{l}\text { Foram incluídos } 1000 \text { indivíduos. A prevalência de HA foi maior nos ho- } \\
\text { mens }(34,6 \%) \text { do que nas mulheres }(27,2 \%) \text {. Entre os hipertensos, } 62,5 \% \\
\text { tinham conhecimento de seu diagnóstico, } 82,4 \% \text { estavam em tratamen- } \\
\text { to e } 60,0 \% \text { tinham pressão arterial controlada. }\end{array}$ \\
\hline
\end{tabular}

dores. Para identificação do diagnóstico, tratamento e controle da HA e ao mesmo tempo a realização do rastreamento contribuem para diminuição de riscos para DCV.

Dessa maneira, com o objetivo de ampliar a conscientização da população e $o$ controle da HA, foi criado em parceria com a Liga Mundial de Hipertensão, em inglês, World Hypertension League (WHL) e a Sociedade Internacional de Hipertensão, em inglês, International Society Hypertension (ISH), o Dia Mundial da Hipertensão, em inglês, "World Hypertension Day" (WHD) "17 de maio", que passou a ser padronizado o Mês de Maio com mês da medição global da pressão arterial ${ }^{(28)}$.

Um dos principais desafios encontrados para se estabelecer uma prevenção mundial, é a padronização dos programas de rastreamentos da pressão arterial. A WHL e a ISH reconhecem que há desafios complexos à detecção precoce, prevenção e controle da HA e propóe o rastreamento deve ser feito a partir de medidas rigorosas da pressão arterial e conscientização sobre DCNT na comunidade ${ }^{(28,29)}$. Estes programas devem ressaltar o rigor junto as etapas do procedimento da medida da PA, utilizando-se de aparelhos validados, conforme preconiza diretrizes nacionais e internacionais ${ }^{(3,29)}$.

Assim, vale ressaltar importantes pontos para realização de programas de rastreamentos da pressão arterial: a equipe de voluntários que será responsável pela coleta de dados precisa ser devidamente capacitada e receber um treinamento padronizado de medição da pressão arterial, o resultado da medida da pressão arterial deverá ser por meio da média das duas últimas medidas. Os participantes deverão receber orientações em saúde, no mínimo sobre aconselhamento dietético e de estilo de vida para reduzir a $\mathrm{PA}^{(23)}$.

Programas de rastreamentos da pressão arterial tem sido realizados para controle e prevenção da $\mathrm{HA}$, na qual fornecem uma intervenção custo-efetiva e endossada pelo WHL para melhorar as taxas de detecção, controle e tratamento da HA, com o objetivo de reduzir a morbidade e a mortalidade cardiovascular ${ }^{(30)}$. Os estudos identificados nesta revisão demostram a efetividade dos programas de rastreamento no mundo.

$\mathrm{Na}$ Índia foi realizado um rastreamento 
utilizando o aparelho eletrônico Omron HEM8712, e conduzida uma única medida por pessoa, onde aqueles que apresentavam valor $\mathrm{PA} \geq 140 \mathrm{mmHg}$ (sistólica) e/ou $\geq 90 \mathrm{mmHg}$ (diastólica), eram informados a realizarem acompanhamento médico, já que uma única leitura não é passível de diagnóstico de HA. Os resultados desse estudo mostraram que 5760 pessoas foram rastreadas, dessas 1783 apresentavam HA, dos quais 1580 desconheciam o diagnóstico. As baixas taxas de conscientização, tratamento e controle da HA ressaltam o desafio constante do rastreamento e tratamento da HA na Índia ${ }^{(16)}$.

Rastreamento desenvolvido nos Estados Unidos, realizou um rastreamento que buscou identificar importantes variáveis da medida da PA como $(\geq 120 / 80 \mathrm{mmHg}$; $\geq 140$ / 90 e $\geq 160$ / 100 mm Hg) utilizando um dispositivo de alta precisão BpTRU, este aparelho desconta a medição inicial e leva uma média dos últimos cinco. No rastreamento de 354 pacientes, pode-se verificar que dois terços de pacientes sem diagnóstico de HA apresentaram PA acima do limiar de $120 / 80 \mathrm{mmHg}$ em rastreamento com uso do aparelho BpTRU. Foi feita a escolha do braço com valor maior de PA, de acordo com as instruções contidas no manual do BpTRU, e realizada a sequência de 5 medidas com intervalo de 1 minuto, levando a média das medições ${ }^{(17)}$.

Outro rastreamento realizado globalmente padronizado utilizando esfigmomanômetros digitais (OMRON), e por meio dessa campanha foi possível identificar que mais de um quarto $(33,4 \%)$ dos participantes eram hipertensos tendo mais da metade (59,5\%) cientes de seu diagnóstico e $55,3 \%$ faziam tratamento medicamentoso. Ainda, foram identificados 335.000 hipertensos não tratados ou tratamentos inadequados ${ }^{(18)}$.

Em estudo realizado em Malawi foram analisados 3 programas de rastreamento de DCNT com foco em HA e diabetes em um sistema público de saúde, com pessoas que estão aguardando atendimentos ambulatoriais e em uma clínica. Essa vinculação do serviço já prestado, o ambulatorial ou clíni$\mathrm{ca}$, com o programa de rastreamento mos- trou-se viável e com resultados positivos no foco da proximidade do usuário com o serviço de saúde ${ }^{(21)}$.

Em rastreamento realizado na China com 3899 pessoas idosas sem diagnóstico prévio de HA, foram treinados profissionais para realizarem a medição com esfigmomanômetro de mercúrio no braço direito, utilizando assim um padrão de rastreamento. Foram instruídos a realizarem acompanhamento médico e mudarem hábitos de vida em busca da redução da PA aqueles que apresentaram valores alterados durante a medição domiciliar, comprovando em dois anos que ações como essas trazem impacto positivo a longo prazo $^{(22)}$.

\section{A realização de}

rastreamento e

busca por doentes

sem conhecimento

de seu diagnóstico

é uma maneira

de prevenção de complicações oriundas de DCNT.

Um estudo realizado em Israel criou uma estação em um ambiente público com pessoas não hospitalizadas mostrou a eficácia desse serviço pensando que a medicina preventiva nem sempre consegue atingir seus propósitos por não haver busca pelo serviço de saúde. Os principais objetivos dessa estação eram: a realização de rastreamento de disglicemia, diabetes e alterações na pressão arterial; aproximação da comunidade local com o serviço de saúde de for- ma mais informal. Cuidados preventivos de saúde são importantes na prática de saúde e podem trazer melhorias significativas à população ${ }^{(20)}$.

$\mathrm{Na}$ Itália também utilizou da técnica de ambiente extra hospitalar, porém com intuito de avaliar a prevalência de HA e outras DCV, trazendo o resultado já esperado onde pacientes hipertensos possuem maior risco cardiovascular ${ }^{(24)}$.

Na Suíça em 2015 foi desenvolvido um estudo que mostrou que a utilização de espaços extra hospitalares se tornam de fato eficazes no rastreamento de hipertensos. Portanto, pode-se chegar a conclusão de que a elaboração de novas estratégias pensadas fora do ambiente hospitalar, que atingissem hipertensos não diagnosticados ou com tratamento incorreto fossem desenvolvidas, além do foco na prevenção de demais complicações advindas dessas doenças ${ }^{(26)}$.

A realização de rastreamento e busca por doentes sem conhecimento de seu diagnóstico é uma maneira de prevenção de complicações oriundas de DCNT. Para alcançar isso foi proposto em Johannesburg a implementação de um projeto que realizava o rastreamento em visitas domiciliares em um subúrbio. Ao final foi constatado que um menor número de pessoas do que o esperado buscou o serviço de saúde para confirmação diagnóstica de HA e hiperglicemia, o que mostra uma falha na realização do rastreamento feita inicialmente ${ }^{(25)}$.

No que se refere a medida da PA, estudo aponta a importância da medição da PA em ambos os braços, uma vez que uma diferença anormal entre os braços na PA está associada a um risco aumentado de anormalidades vasculares e DCV. A medição em braço duplo, não apenas auxilia a evidenciar potenciais fatores de risco cardiovasculares, mas também pode melhorar o diagnóstico de HA em geral. E tal medida deve ser realizada de forma adequada para obtenção de resultados fidedignos ${ }^{(15,31)}$.

No presente estudo, $10 \%$ dos indivíduos pareciam ter pressão arterial elevada em um braço (geralmente o braço dominante), mas não no outro. Assim concluíram que a não realização da medição do braço duplo pode, portanto, levar a uma falha na detec- 


\section{artigo}

Costa, B.C.P.; Hilario, V.G.; Veiga, E.V.

Programas de rastreamentos dos valores da pressão arterial no mundo: revisão integrativa

ção de importantes fatores de risco cardiovascular, incluindo o diagnóstico de $\mathrm{HA}^{(15)}$.

Uma pesquisa para determinar a eficácia de intervenção sobre pressão arterial, colesterol e educação em DCV em calouros da faculdade, realizou um rastreamento da PA, na qual os 25 participantes tiveram a pressão arterial medida três vezes no braço não dominante com um monitor digital de pressão arterial validado por HEM-907X e manguito de tamanho adequado. A PA sistólica e diastólica média foi calculada a partir das duas últimas medições ${ }^{(32)}$.

Foram realizadas três visitas no estudo, a qual a primeira consistia em responder questionários, fornecer uma amostra de sangue e medir a pressão arterial. A segunda visita foi uma sessão de conscientização educacional e a terceira foi uma repetição da primeira, que ocorreu aproximadamente dois meses após a primeira visita. Ao final do estudo, identificaram a importância da educação em saúde e a mudanças de hábitos para redução da pressão arterial e colesterol, pois estes resultaram fortes fatores de risco para $\mathrm{DCV}^{(32)}$.

No Brasil, uma pesquisa que avalia a adesão e qualidade do tratamento para HA de forma comparativa entre homens e mulheres, mostrou que apesar da prevalência da doença ser maior em pessoas do sexo masculino, pessoas do sexo feminino fazem parte do grupo de maior adesão e busca por conhecimento do seu diagnóstico e controle da doença ${ }^{(27)}$.

Um dos principais passos para que pessoas procurem o serviço de saúde é a conscientização da HA, e então só assim possam obter um controle e mudanças no estilo de vida $^{(22,33)}$. Estudos sugerem que programas de rastreamento podem melhorar a detecção e contribuir para o controle e prevenção da HA. No entanto, para adequada abordagem do rastreamento é necessário um sistema eficaz para garantir um acompanhamento adequado se for detectada pressão arterial alterada ${ }^{(20,34)}$. Programas de rastreamento de HA podem permitir investigações exclusivas nos níveis nacional, regional, étnico e global relacionadas à medição clínica da pressão arterial(31).

\section{CONCLUSÃO}

Programa de rastreamento da pressão arterial é uma estratégia de baixo custo e elevada eficácia para identificar de forma precoce a HA, prevenir e controlar valores elevados de pressão arterial, seja em países desenvolvidos e em desenvolvimento.

O estudo permitiu uma exploração sobre rastreamentos da HA em vários países e cada qual evidenciou a importância de rastrear valores elevados da pressão arterial, para trabalhar na prevenção, controle e conscientização da HA e sobre seus fatores de risco.

Sugere-se novos estudos na temática, para que mais programas de rastreamentos da HA possam ser realizados e desta forma um número maior de pessoas rastreadas e conscientizadas, para diminuir os riscos cardiovasculares.

\section{REFERÊNCIAS}

1. Christofoletti M, Del Duca GF, Gerage AM, Malta DC. Simultaneidad de enfermedades crónicas no transmisibles en 2013 en las capitales brasileñas: prevalencia y perfil demográfico. Epidemiol. Serv. Saúde. 2020;29(1). Doi: 10.5123/S167949742020000100006

2. Malta DC, Bernal RTI, Lima MG, Araújo SSC, Silva MMA, Freitas MIF. et al. Doenças crônicas não transmissíveis e a utilização de serviços de saúde: análise da Pesquisa Nacional de Saúde no Brasil. Rev. Saúde Públ. 2017b; 51(Supl 1):4s. Doi:10.1590/ s1518-8787.2017051000090

3. Sociedade Brasileira de Cardiologia. $7^{\text {a }}$ Diretriz Brasileira de Hipertensão Arterial. Arq Bras Cardiol [Internet]. 2016 [citado 2018 Dez 06]. 107(3Supl.3):1-83.Disponível em: http://publicacoes.cardiol.br/2014/diretrizes/2016/05_HIPERTENSAO ARTERIAL.pdf

4. Dias EG, Almeida FG, Caires HLD, Santos TAS, Antunes Jorge S, Mishima SM. Evaluation of a Family Health Strategy about the promotion of adherence to treatment and control of hypertension under the optics of the elderly. J Health Sci Inst [Internet]. 2016 [cited 2020 jan 06];34(2):88-92. Available from: https:// www.unip.br/presencial/comunicacao/publicacoes/ics/edicoes/2016/02_abr-jun/V34_n2_2016_p88a92.pdf

5. Oliveira TMF, Almeida TCF. Adequação do manguito durante a medida da pressão arterial: uma revisão integrativa. Ciência e Saúde, 8(1):35-41, 2015. Doi: 10.15448/1983-

\section{$652 X .2015 .1 .19419$}

6. Nerenberg KA, Zarnke KB, Leung AA, Dasgupta K, Butalia S, McBrien K. et al. Hypertension Canada Guidelines for Diagnosis, Risk Assessment, Prevention, and Treatment of Hypertension in Adults and Children. Canadian Journal of Cardiology, 34(5):50625, 2018.

7. Seabra CAM, Xavier SPL, Sampaio YPCC, Oliveira MF, Quirino GS, Machado MFAS. Health education as a strategy for the promotion of the health of the elderly: an integrative review. Rev. Bras. Geriatr. Gerontol. 2019;22(4) Doi:10.1590/198122562019022.190022

8. Rêgo AS, Laqui VS, Trevisan FG, Jaques AE, Oliveira RR, Radovanovic, CAT. Factors associated with inappropriate blood pressure in hypertensive patients. Cogitare Enferm. 2018; 1(23):e54087. Doi: $10.5380 /$ ce.v23i1.54087

9. Mendes KDS, Silveira RCCP, Galvão CM. Revisão integrativa: método de pesquisa para a incorporação de evidências na saúde e na enfermagem. Texto Contexto Enferm 2008; 17(4):758-64. Doi: 10.1590/S0104-07072008000400018.

10. Considine J; Shaban RZ, Fry M, Curtis K. Evidence based emergency nursing: designing a research question and searching the literature. International Emergency Nursing. 2017; 32:7882. doi:10.1016/j.ienj.2017.02.001.

11. Ouzzani $M$, Hammady $H$, Fedorowicz Z, Elmagarmid A. 


\section{REFERÊNCIAS}

Rayyan - a web and mobile app for systematic reviews. Syst Rev. 2016;5:210.

12. Galvão TF, Pansani TSA. Principais itens para relatar Revisões sistemáticas e Meta-análises: A recomendação Prisma. Epidemiol. Serv. Saúde. 2015;24(2). Doi:10.5123/S167949742015000200017

13. Souza MT, Silva MD, Carvalho R. Integrative review: what is it? How to do it? einstein. 2010; 8(1 Pt 1):102-6

14. Melnyk BM, Fineout-Overholt E. Evidence-based practice in nursing and healthcare. A guide to best practice. 2 edition Philadelphia:Wolters Kluwer, Lippincott Williams and Wilkins, 2011.

15. Omboni S, Verberk WJ. Simultaneous double arm automated blood pressure measurement for the screening of subjects with potential vascular disease: a community study. Blood Press. 2019;28(1):15-22. doi: 10.1080/08037051.2018.1539619.

16. Balsari S, Vemulapalli P, Gofine M, Oswal K, Merchant R, Saunik $\mathrm{S}$. et al. A retrospective analysis of hypertension screening at a mass gathering in India: implications for non-communicable disease control strategies. J Hum Hypertens. 2017;31(11):750753. doi: 10.1038/jhh.2017.54.

17. Goldberg E. M. , Wilson T, Saucier C, Brody AM, Levy PD, Eaton CB. et al. Achieving the BpTRUth: emergency department hypertension screening and the Centers for Medicare \& Medicaid Services quality measure. J Am Soc Hypertens. 2017;11(5):290294. doi: 10.1016/j.jash.2017.03.003.

18. Beaney T, Schutte AE, Tomaszewski M, Ariti C, Burrell LM, Castillo RR. et al. May Measurement Month 2017: an analysis of blood pressure screening results worldwide. The Lancet. 2018; 6(7):E736-E743. Doi: 10.1016/S2214-109X(18)30259-6

19. Bar-Dayan Y, Boaz M, Landau Z, Zeev F, Jakubowicz D, Wainstein J. Using a public health station for screening of undiagnosed dysglycemia and hypertension Prim Care Diabetes. 2016 Oct;10(5):324-8. Doi: 10.1016/j.pcd.2016.02.001.

20. Handler J, Mohan Y, Kanter MH, Reynolds K, Li X, Nguyen M. et al. Screening for High Blood Pressure in Adults During Ambulatory Nonprimary Care Visits: Opportunities to Improve Hypertension Recognition. I Clin Hypertens (Greenwich).2015;17(6):431-9. Doi: 10.1111/jch.12517.

21. Kachimanga C, Cundale K, Wroe E, Nazimera L, Jumbe A, Dunbar $\mathrm{E}$. et al. Novel approaches to screening for noncommunicable diseases: Lessons from Nono, Malawi. Malawi Med J. 2017;29(2):78-83. doi: 10.4314/mmj.v29i2.1.

22. Chen S, Sudharsanan N, Huang F, Liu Y, Geldsetzer P, Bärnighausen T. Impact of community based screening for hypertension on blood pressure after two years: regression discontinuity analysis in a national cohort of older adults in China. BMJ. 2019; 366. Doi: 10.1136/bmj.14064

23. Poulter NR, Schutte $A E$, Tomaszewski M, Lackland DT. May Measurement Month: a new joint global initiative by the International Society of Hypertension and the World Hypertension League to raise awareness of raised blood pres- sure. J Hypertens. 2017 May;35(5):1126-1128. doi: 10.1097/ HJH.0000000000001346.

24. Destro M, Dognini GP, Pozzi A, Cagnoni F, Galimberti VC, C Cavalleri. et al. Hypertension and cardiovascular risk factors: a shot on northern Italy population in real life setting. J Hypertens. 2015; 33(Suppl 1). Doi: 10.1097/01.hjh.0000467536.84037. ae

25. Rheeder P, Muthembe T, Lawson S, Brink J. Diabetes and hypertension in Zandspruit, Johannesburg 2012-2014. J South African Family Practice. 2016;58(6):219-224. Doi:10.1080/207 86190.2016.1198089

26. Risse J, Laurière, E, Iraqi $M$, Fay $R$, Burnier $M$, Boivin JM . Screening for Hypertension in the Barbershop: A Franco-Moroccan feasibility study (The DECOIFFA study). J Hypertens. 2015; 33:e69. Doi: 10.1097/01.hjh.0000467534.06908.a5

27. Jardim T, Neiva T, Rodrigues R, Arantes A, Barros C, Chinem B. et al. Diferences in prevalence, awareness, tratament and control rates of hypertension between male and female. J Hypertens. 2015;33:e69. Doi: 10.1097/01.hjh.0000467535.06908.ec

28. Campbell NRC, Lackland DT, Lisheng L, Zhang XH, Peter M. Nilsson, Niebylski ML. et al. The World Hypertension League: where now and where to in salt reduction. Cardiovasc Diagn Ther. 2015; 5(3): 238-242. Doi: 10.3978/j.issn.22233652.2015.04.08

29. Nerenberg KA, Zarnke KB, Leung AA, Dasgupta K, Butalia S, McBrien K. et al. Hypertension Canada's 2018 Guidelines for Diagnosis, Risk Assessment, Prevention, and Treatment of Hypertension in Adults and Children. Canadian Journal of Cardiology. 2018;34(5):506e525. Doi: 10.1016/j.cjca.2018.02.022

30. Mangat BK, Campbell N, Mohan S, Niebylski ML, Khalsa TK, Berbari AE et al. Resources for Blood Pressure Screening Programs in Low Resource Settings: A Guide From the World Hypertension League. J Clin Hypertens. 2015;17(6) :418-20. Doi: 10.1111/jch.12499.

31. Destefano RM, Schmitt FRA, Starke S, Santa Helena ET. Adequacy of sphygmomanometer cuff to brachial circumference of people attended in Primary Health Care Centers. Rev B ras Epidemiol. 2017; 20(1):81-90. Doi: 10.1590/19805497201700010007.

32. Melnyk J, Panza G, Zaleski A, Taylor B. Awareness and Knowledge of Cardiovascular Risk Through Blood Pressure and Cholesterol Testing in College Freshmen. Am J Health. 2015;46(3). doi: 10.1080/19325037.2015.1023474.

33. Oliveira PTG, Almeida JM. Healthy living group: lifestyle change analysis of patients with systemic arterial hypertension and type 2 diabetes. Rev Fac Ciênc Méd Sorocaba. 2018;20(3):142-9. Doi: 10.23925/1984-4840.2018v20i3a5

34. Beaney T, Schutte AE, Stergiou GS, Borghi C, Burger D, Charchar FJ. et al. May Measurement Month 2019: The Global Blood Pressure Screening Campaign of the International Society of Hypertension. Hypertension. 2020. Doi: 10.1161/HYPERTENSIONAHA.120.14874 